\title{
Intracranial Stenting After Failed Thrombectomy in Patients With Moderately Severe Stroke: A Multicenter Cohort Study
}

\begin{abstract}
Lukas Meyer $^{1 *}$, Jens Fiehler ${ }^{1}$, Götz Thomalla ${ }^{2}$, Lars Udo Krause ${ }^{3}$, Stephan Lowens ${ }^{4}$, Jan Rothaupt ${ }^{5}$, Byung Moon Kim ${ }^{6}$, Ji Hoe Heo ${ }^{7}$, Leonard $\mathrm{YeO}^{8,9}$, Tommy Andersson ${ }^{8,10}$, Christoph Kabbasch ${ }^{11}$, Franziska Dorn ${ }^{12}$, René Chapot ${ }^{5}$, Christian Paul Stracke ${ }^{5 \dagger}$ and Uta Hanning ${ }^{1+}$
\end{abstract}

\begin{abstract}
1 Department of Diagnostic and Interventional Neuroradiology, University Medical Center Hamburg-Eppendorf, Hamburg, Germany, ${ }^{2}$ Department of Neurology, University Medical Center Hamburg-Eppendorf, Hamburg, Germany, ${ }^{3}$ Department of Neurology, Klinikum Osnabrück, Osnabrück, Germany, ${ }^{4}$ Department of Radiology, Klinikum Osnabrück, Osnabrück, Germany, ${ }^{5}$ Department of Intracranial Endovascular Therapy, Alfried-Krupp Hospital, Essen, Germany, ${ }^{6}$ Department of Radiology, Interventional Neuroradiology, Severance Stroke Center, Severance Hospital, Yonsei University College of Medicine, Seoul, South Korea, ${ }^{7}$ Department of Neurology, Severance Stroke Center, Severance Hospital, Yonsei University College of Medicine, Seoul, South Korea, ${ }^{8}$ Department of Neuroradiology, Karolinska Institutet, Karolinska University Hospital and Clinical Neuroscience, Stockholm, Sweden, ${ }^{9}$ Division of Neurology, Department of Medicine, National University Health System, Singapore, Singapore, ${ }^{10}$ Department Medical Imaging, AZ Groeninge, Kortrijk, Belgium, ${ }^{11}$ Department of Neuroradiology, University of Cologne, Cologne, Germany, ${ }^{12}$ Department of Neuroradiology, University Hospital of Munich, Munich, Germany
\end{abstract}

Background and Purpose: Recently, acute intracranial stenting (ICS) has gained more interest as a potential bailout strategy for large vessel occlusions (LVO) that are refractory to thrombectomy. However, there are currently no reports on ICS in patients with moderately severe stroke discussing the question if implementing a permanent stent is feasible and leads to improved recanalization after failed thrombectomy.

Methods: We analyzed a large multicenter database of patients receiving ICS for anterior circulation LVO after failed thrombectomy. Inclusion criteria were defined as: Moderately severe stroke (National Institute Health Stroke Scale (NIHSS) $\leq 9$ on admission), anterior circulation LVO, acute ICS after failed stent retriever MT. Primary endpoint was the rate of improved successful recanalization after ICS defined as a modified Thrombolysis In cerebral Infarction (mTICl) score $\geq 2 b$. Favorable neurological outcome was defined as an early neurological improvement (ENI) of 4 points or reaching 0 with respect to baseline NIHSS.

Results: Forty-one patients met the inclusion criteria. A median of 2 retrievals were performed (IQR 1-4) prior decision-making for ICS. ICS led in $90.2 \%(37 / 41)$ of cases to a final $\mathrm{mTICl} \geq 2 \mathrm{~b}$ with significant improvement $(p<0.001)$ after the last retrieval attempt. The median NIHSS decreased $(p=0.178$ ) from 7 (IQR 3.5-8) on admission to 2.5 (IQR 0-8.25) at discharge. ENI was observed in 47.4\% (18/38). sICH occurred in 4.8\% (2/41).

Conclusion: ICS after failed thrombectomy appears to effectively improve recanalization rates in patients with moderately severe strokes. Thus, ICS should be considered also for patients with baseline NIHSS $\leq 9$ if thrombectomy fails.

Keywords: failed thrombectomy, stroke, intracranial stenosis, stent, ICAD 


\section{INTRODUCTION}

Mechanical thrombectomy (MT) has become the standard of care for acute ischemic stroke due to large vessel occlusions (LVO). However, up to $29 \%$ of all mechanical thrombectomies (MT) fail due to several conditions, such as intracranial atherosclerotic disease (ICAD), calcified wall-adherent thrombi, dissections, or other rare pathologies (1-3). Accordingly, the best currently available evidence for the endovascular treatment of ICAD is based on the SAMMPRIS and the VISSIT study $(4,5)$ showing the superiority of best medical treatment over elective intracranial stenting. Recently, acute intracranial stenting (ICS) has been reported to be a highly promising bailout strategy for theses frustrating thrombectomy cases with predictably poor outcomes (6-11). Since these cases are still rare, past retrospective studies mostly analyzed heterogeneous cohorts, including a wide range of stroke severities (12).

It has been suggested that a low NIHSS (National Institute Health Stroke Scale) score is a more frequent observation in patients with acute occlusions of preexisting ICAD, presumably based on adaptation of collaterals to the chronic low flow conditions $(13,14)$. However, these patients arriving with comparably low NIHSS scores could still have a poor prognosis and high risk for stroke recurrence without sufficient therapy $(15,16)$. Therefore, it might not be reasonable to base decisionmaking for bailout strategies after failed thrombectomy on patients' initial NIHSS score only.

This study analyzes ICS for patients with moderately severe anterior circulation LVO strokes (NIHSS score $\leq 9$ upon admission) to better estimate potential risks and benefits. We hypothesize that in this patient subgroup, ICS is a feasible bailout strategy to achieve improved vessel recanalization after failed thrombectomy.

\section{METHODS}

\section{Patient Selection}

We analyzed all patients with moderately severe stroke from a large international ICS multicenter cohort $(n=4751)$ treated between $01 / 2014$ and $12 / 2018$. ICS after failed thrombectomy was performed in 210 cases with a relative frequency of 4.4 $\%(210 / 4751)$ in relation to all thrombectomies performed within the study period. Inclusion criteria were: (1) Moderately severe stroke (admission NIHSS $\leq 9$ ), (2) anterior circulation LVO (3) MT performed exclusively with stent retrievers (4) acute intracranial stenting as a bailout strategy (Figure 1). The study was approved by the local ethics committee (Chamber of Physicians, Hamburg, Germany). Due to the retrospective and anonymized study design, informed consent of the patients was not required. Some data were part of previously published cohorts $(7,11)$.

Abbreviations: ASPECTS, Alberta Stroke Program Early CT Score; ENI, Early Neurological Improvement; LVO, Large Vessel Occlusion; MT, Mechanical Thrombectomy; mTICI, Modified Thrombolysis In cerebral Infarction; NIHSS, National Institute Health Stroke Scale; ICS, Intracranial Stenting; ICAD, Intracranial Atherosclerotic Disease; IVT, Intravenous Thrombolysis; sICH, symptomatic Intracerebral Hemorrhage.

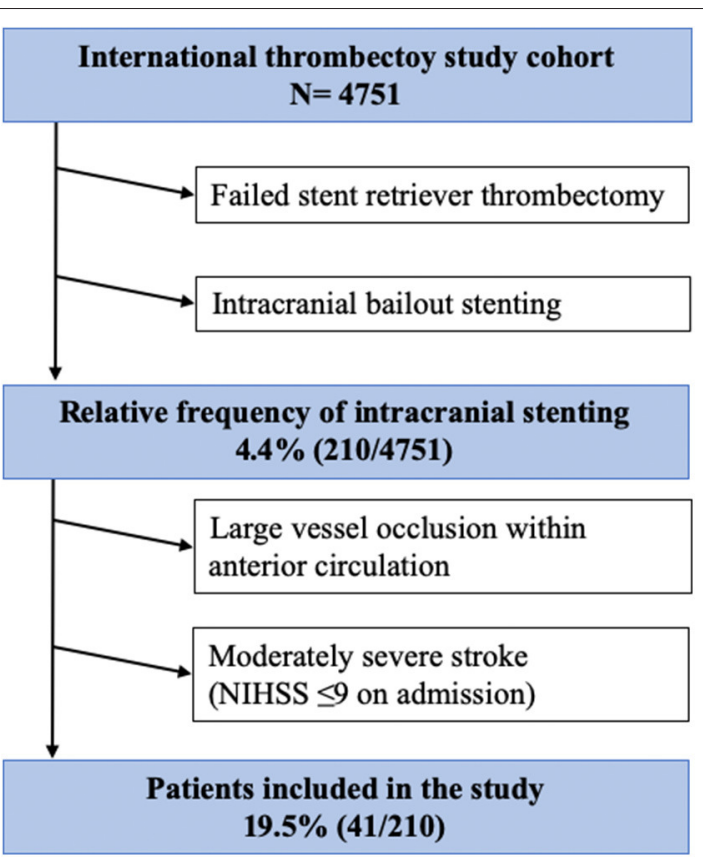

FIGURE 1 | Flow chart of patient inclusion.

\section{Baseline Characteristics}

Available baseline characteristics were analyzed (Table 1). Early ischemic changes were graded with the Alberta Stroke Program Early CT Score (ASPECTS) on non-contrast computed tomography. Experienced neurologists examined all patients applying the NIHSS on admission and discharge. If eligible, all patients received intravenous thrombolysis (IVT) prior to MT.

\section{Intervention}

In all cases MT performed with approved stent retriever devices did not lead to sufficient recanalization or direct reocclusion after thrombectomy occurred. Accordingly, the number of retrieval attempts as well as the moment of decision-making for ICS and declaring thrombectomy as failed was left to the interventionalist. All types of stent retriever, the number of thrombectomy maneuvers, as well as the stent design (balloon or self-expanding) were evaluated. The recanalization result was evaluated with the modified thrombolysis in cerebral infarction (mTICI) score.

\section{Procedural and Functional Outcome}

Primary endpoint was the rate of improved recanalization after ICS assessed by the rate of successful recanalization defined as $\mathrm{mTICI} \geq 2 \mathrm{~b}$. Neurological outcome was assessed by the rate of early neurological improvement (ENI) defined as a decrease in NIHSS at discharge from baseline of at least 4 points as previously described (17) or reaching 0 . The rate of favorable functional outcome was assessed as $\mathrm{mRS} \leq 2$ at 90 days. Due to the retrospective approach, ENI data for 3 patients and 90 day mRS data for 14 patients were missing. For safety assessment, cases with symptomatic intracranial hemorrhage ( $\mathrm{sICH}$ ) according to ECASS-II (18), mortality, and intervention related complications 
TABLE 1 | Overview of patients' baseline characteristics.

\begin{tabular}{lc}
\hline Baseline characteristics & Study cohort $(\boldsymbol{n}=\mathbf{4 1}$ \\
\hline Clinical and imaging & \\
Age (years), median (IQR) & $64(48-73)$ \\
Sex (men) \% (n) & $70.7(29 / 41)$ \\
ASPECTS, median (IQR) & $8(8-10)$ \\
NIHSS on admission, median (IQR) & $7(3.5-8)$ \\
Pre-stroke mRS, \% (n) & \\
0 & $65.9(27 / 41)$ \\
1 & $26.8(11 / 41)$ \\
2 & $4.9(2 / 41)$ \\
3 & $2.4(1 / 41)$ \\
Target vessel, $n$ (\%) & \\
ICA & $36.6(15 / 41)$ \\
M1 & $53.6(22 / 41)$ \\
M2 & $9.8(4 / 41)$ \\
Procedural & \\
Intravenous thrombolysis, \% (n) & $61(25 / 41)$ \\
CT to groin-puncture (minutes), median (IQR) & $104(74.0-148)$ \\
Type of stent, \% (n) & \\
\hline
\end{tabular}

were evaluated. Further, the antiplatelet therapy regimes were recorded and analyzed.

\section{Statistical Analysis}

Continuous variables are displayed as mean with SD or median with interquartile range. For categorical data, absolute, and relative frequencies are displayed. Wilcoxon test was performed to compare stroke severity on admission and discharge, as well as recanalization status before and after stenting. $P \leq 0.05$ were considered significant. Analyses were performed using SPSS V.25 (IBM Corporation, Armonk, New York, USA).

\section{RESULTS}

\section{Baseline Characteristics}

$19.5 \%(41 / 210)$ of all patients in the multicenter ICS database met the required inclusions criteria. Median age was 64 years (IQR 48.5-73.5) and 70.7\% (29/41) were men. On admission median ASPECTS was 8 (IQR 8-10) and median 7 (IQR 3.58 ), whereas the median pre-stroke mRS was 0 (IQR $0-1$ ). $39 \%$ $(16 / 41)$ of the patients received IVT prior to MT. Target vessels for ICS were ICA in 36.6\% (15/41), MCA M1 in 53.7\% (22/41) and MCA M2 in $9.8 \%$ (4/41). A self-expandable stent (Acclino flex ${ }^{\circledR}$, Neuroform ${ }^{\circledR}$, Solitaire ${ }^{\circledR}$, Enterprise ${ }^{\circledR}$, Wingspan ${ }^{\circledR}$ ) was utilized in all cases $(100 \%, 41 / 41)$ for bailout ICS. Information on periprocedural antithrombotic medication was available in $51.1 \%(21 / 41)$. In $14.3 \%(3 / 21)$ IV acetylsalicylic acid only and in $85.7 \%(18 / 21)$ glycoprotein-IIb/IIIa-antagonists were administered. Post-interventionally, all patients received dual antiplatelet therapy for 3 months. Table 1 gives an overview of patients' baseline characteristics.

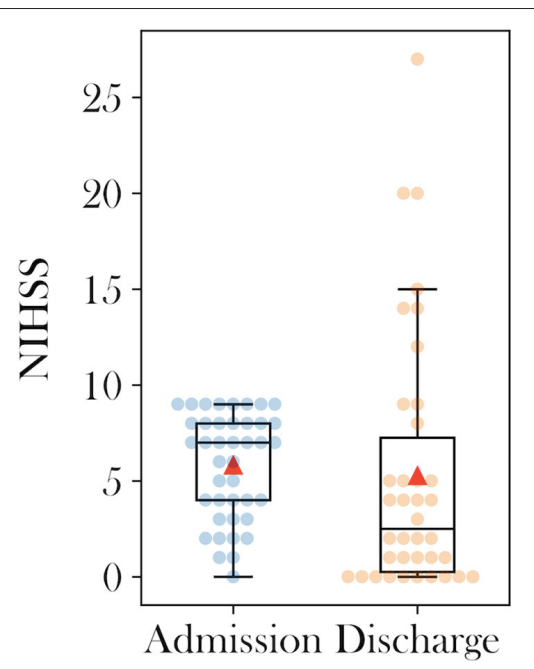

FIGURE 2 | Admission and discharge boxplots of National Institutes of health stroke scale scores.

TABLE 2 | Overview of procedural results, neurological and functional outcome, complications, and mortality.

\begin{tabular}{lc}
\hline Results & All patients $(\boldsymbol{n}=\mathbf{4 1})$ \\
\hline Procedural & $2(1-4)$ \\
Passes of retriever, median (IQR) & $43.9(18 / 41)$ \\
Successful recanalization after last pass & \\
(mTICl 2b/3), $n$ (\%) & $90.2(37 / 41)$ \\
Successful recanalization after ICS & \\
(mTICl 2b/3), $n$ (\%) & \\
Neurological improvement & $2.5(0-8.25)$ \\
NIHSS discharge, median (IQR) & $47.4(18 / 38)$ \\
ENI at discharge \% (n) & \\
Complication & $4.8(2 / 41)$ \\
sICH \% (n) & \\
Functional outcome & \\
mRS $\leq 2$ at 90 days \% (n) & $74.1(20 / 27)$ \\
Mortality & \\
At 90 days \% (n) & $2.7(1 / 27)$ \\
\hline
\end{tabular}

\section{Procedural and Functional Outcome}

A median of 2 MT maneuvers were performed (IQR 1-4) prior to ICS. After the final MT attempt $\mathrm{mTICI} \geq 2 \mathrm{~b}$ was achieved in $43.9 \%$ $(18 / 41)$. Acute ICS significantly increased $(p<0.001)$ the rate of $\mathrm{mTICI} \geq 2 \mathrm{~b}$ to $90.2 \%$ (37/41). The median NIHSS decreased from 7 (IQR 3.5-8) on admission to 2.5 (IQR 0-8.25) at discharge (Figure 2) without reaching statistical significance $(p=0.178)$. ENI was observed in $47.4 \%(18 / 38)$ and sICH occurred in $4.8 \%$ $(2 / 41)$ of all patients. At 90 days the rate of $\mathrm{mRS} \leq 2$ was $74.1 \%$ (20/27; Table 2$)$ and the mortality was $2.7 \%(1 / 27)$.

\section{DISCUSSION}

Recent retrospective case series have suggested reconsidering ICS as a bailout strategy when MT fails to recanalize LVOs 
$(11,19)$. All these ICS studies report on typical thrombectomy cohorts with a median admission NIHSS ranging from 14 to 19 (12). However, there are currently no reports on ICS after failed thrombectomy for a homogenous group of less severe stroke patients. Accordingly, we defined strokes with a baseline NIHSS $\leq 9$ on admission as moderately severe. This study assesses a potential benefit of ICS after failed thrombectomy for this subgroup.

Successful recanalization is a strong predictor for long-term favorable functional outcome (mRS $\leq 2)$ after MT (20). In cases with ICAD, acute or prolonged reocclusion after MT is a severe complication leading to poor outcomes (21). Even with supposedly higher proportions of low NIHSS on admission in patients with ICAD due to possibly better adapted collateral flow, a sufficient and sustainable vessel recanalization seems crucial to increase chances for long-term favorable outcomes and to prevent stroke recurrence (14). Thus, it is an important finding in our study that ICS increased the rate of successful recanalization leading to $90 \%$ mTICI $\geq 2 \mathrm{~b}$ after failed MT attempts.

ENI was observed in $47 \%$ of the cohort with a median NIHSS decrease of 4.5 points from admission to discharge. This finding suggests a neurological benefit of ICS in our cohort, however the prespecified level of statistical significance was not reached most likely due to the underpowered sample size of the study. Furthermore, the 90 days favorable functional outcome $(\mathrm{mRS} \leq 2)$ was above average $(74.1 \%, 20 / 27)$ of past thrombectomy landmark studies (1). This finding highlights both, the limitations of the retrospective study design with missing follow-up data and the strength of the finding, showing that even if poor outcome had been observed in all 14 missing patients, the final rate of favorable outcome would still have been comparably good with $49 \%$ of $\mathrm{mRS} \leq 2(20 / 41)$.

The necessity of antiplatelet therapy after permanent stenting has always been a major concern in endovascular stroke treatment due to its increased risk for intracerebral bleeding (22). Choosing ICS for patients presenting with low NIHSS might be of even higher concern since the risk-benefit balance is not comparable to a patient with a high baseline NIHSS facing a high risk of poor outcome. In our study on ICS for moderately severe stroke, $2 / 41$ patients experienced $\mathrm{sICH}$. This result is comparable to those in the HERMES meta-analysis with $4.4 \%$ and is unexpectedly low compared to the latest acute ICS studies that reported on sICH rates ranging from 8 to $17 \%(1,12)$. Antithrombotic medication was administered periinterventionally in all our cases, some even in combination with IVT. Even though detailed information were only available in $50 \%$ of all patients, our finding is in line with the latest studies observing the safety of stenting combined with antiplatelet therapy in the setting of acute tandem occlusions $(23,24)$.

\section{REFERENCES}

1. Goyal M, Menon BK, van Zwam WH, Dippel DW, Mitchell PJ, Demchuk AM, et al. Endovascular thrombectomy after large-vessel ischaemic stroke: a metaanalysis of individual patient data from five randomised trials. Lancet. (2016) 387:1723-31. doi: 10.1016/S0140-6736(16)00163-X
Even though we do not have data on stent patency in this cases series due to missing follow-up imaging, Chang et al. (19) previously observed that a favorable 90 day outcome $(\mathrm{mRS} \leq 2)$ is significantly associated with stent patency. The median number of retrieval attempts in our study was 2. Since all cases were performed in tertiary stroke centers with expertise in all kinds of neurointerventions, this finding is consistent with the latest reports on risk-benefit ratio of additional retrieval attempts and encourages to perform acute ICS after a maximum of three thrombectomy maneuvers even in patients with moderately severe strokes $(20,25)$.

\section{LIMITATIONS}

Our study has all limitations that come along with a retrospective study design. Major limitations are the aforementioned missing data on antithrombotic medication and follow-up outcome at 90 days in 14 patients as well as a control group treated with IVT only for comparison of clinical efficacy and safety endpoints.

\section{CONCLUSION}

This study with its focus on moderately severe stroke is in line with recently published articles that suggested the feasibility of ICS as a bailout strategy after failed thrombectomy leading to improved recanalization in the endovascular treatment of acute ischemic stroke.

\section{DATA AVAILABILITY STATEMENT}

The data that support the findings of this study are available from the corresponding author upon reasonable request.

\section{ETHICS STATEMENT}

The studies involving human participants were reviewed and approved by Chamber of Physicians, Hamburg, Germany. Written informed consent for participation was not required for this study in accordance with the national legislation and the institutional requirements.

\section{AUTHOR CONTRIBUTIONS}

LM, UH, CS, and JF: conception and design of the study. LM, UH, GT, CK, LY, FD, TA, BK, SL, JR, JH, and LK: acquisition and analysis. LM, UH, RC, FD, TA, BK, LY, CS, and JF: drafting and revising the manuscript critically.
2. Dobrocky T, Piechowiak E, Cianfoni A, Zibold F, Roccatagliata L, Mosimann P, et al. Thrombectomy of calcified emboli in stroke. Does histology of thrombi influence the effectiveness of thrombectomy? J Neurointerv Surg. (2018) 10:345-50. doi: 10.1136/neurintsurg-2017-013226

3. Yaghi S, Prabhakaran S, Khatri P, Liebeskind DS. Intracranial atherosclerotic disease. Stroke. (2019) 50:1286-93. doi: 10.1161/STROKEAHA.118.024147 
4. Chimowitz MI, Lynn MJ, Derdeyn CP, Turan TN, Fiorella D, Lane BF, et al. Stenting versus aggressive medical therapy for intracranial arterial stenosis. $N$ Engl J Med. (2011) 365:993-1003. doi: 10.1056/NEJMoa1105335

5. Zaidat OO, Fitzsimmons BF, Woodward BK, Wang Z, Killer-Oberpfalzer $\mathrm{M}$, Wakhloo A, et al. Effect of a balloon-expandable intracranial stent vs medical therapy on risk of stroke in patients with symptomatic intracranial stenosis: the VISSIT randomized clinical trial. JAMA. (2015) 313:1240-8. doi: 10.1001/jama.2015.1693

6. Baek JH, Kim BM, Kim DJ, Heo JH, Nam HS, Yoo J. Stenting as a rescue treatment after failure of mechanical thrombectomy for anterior circulation large artery occlusion. Stroke. (2016) 47:2360-3. doi: 10.1161/STROKEAHA.116.014073

7. Cornelissen SA, Andersson T, Holmberg A, Brouwer PA, Söderman $\mathrm{M}$, Bhogal $\mathrm{P}$, et al. Intracranial stenting after failure of thrombectomy with the emboTrap ${ }^{\circledR}$ device. Clin Neuroradiol. (2018) 29:677-683. doi: $10.1007 / \mathrm{s} 00062-018-0697-\mathrm{x}$

8. Jia B, Feng L, Liebeskind DS, Huo X, Gao F, Ma N, et al. Mechanical thrombectomy and rescue therapy for intracranial large artery occlusion with underlying atherosclerosis. J NeuroInterv Surg. (2018) 10:746-50. doi: 10.1136/neurintsurg-2017-013489

9. Zhou T, Li T, Zhu L, Wang M, He Y, Shao Q, et al. Intracranial stenting as a rescue therapy for acute ischemic stroke after stentriever thrombectomy failure. World Neurosurg. (2018) 120:e181-7. doi: 10.1016/j.wneu.2018.08.002

10. Nappini S, Limbucci N, Leone G, Rosi A, Renieri L, Consoli A, et al. Bail-out intracranial stenting with Solitaire $A B$ device after unsuccessful thrombectomy in acute ischemic stroke of anterior circulation. J Neuroradiol. (2019) 46:141-7. doi: 10.1016/j.neurad.2018.05.004

11. Stracke CP, Meyer L, Fiehler J, Leischner H, Bester M, Buhk JH, et al. Intracranial bailout stenting with the Acclino (Flex) Stent/NeuroSpeed Balloon Catheter after failed thrombectomy in acute ischemic stroke: a multicenter experience. J NeuroInterv Surg. (2019) 12:43-7. doi: 10.1136/neurintsurg-2019-014957

12. Wareham J, Flood R, Phan K, Crossley R, Mortimer A. A systematic review and meta-analysis of observational evidence for the use of bailout selfexpandable stents following failed anterior circulation stroke thrombectomy. J Neurointerv Surg. (2018) 11:675-82. doi: 10.1136/neurintsurg-2018-0 14459

13. Lee JS, Lee SJ, Yoo JS, Hong JH, Kim CH, Kim YW, et al. Prognosis of acute intracranial atherosclerosis-related occlusion after endovascular treatment. $J$ Stroke. (2018) 20:394-403. doi: 10.5853/jos.2018.01627

14. Kang DH, Yoon W. Current opinion on endovascular therapy for emergent large vessel occlusion due to underlying intracranial atherosclerotic stenosis. Korean J Radiol. (2019) 20:739-48. doi: 10.3348/kjr.2018.0809

15. Kim JT, Park MS, Chang J, Lee JS, Choi KH, Cho KH. Proximal arterial occlusion in acute ischemic stroke with low NIHSS scores should not be considered as mild stroke. PLoS ONE. (2013) 8:e70996. doi: 10.1371/journal.pone.0070996

16. Mazya MV, Cooray C, Lees KR, Toni D, Ford GA, Bar M, et al. Minor stroke due to large artery occlusion. When is intravenous thrombolysis not enough? Results from the SITS international stroke thrombolysis register. Eur Stroke J. (2018) 3:29-38. doi: 10.1177/2396987317746003

17. Kharitonova T, Mikulik R, Roine RO, Soinne L, Ahmed N, Wahlgren N. Association of early national institutes of health Stroke. Scale improvement with vessel recanalization and functional outcome after intravenous thrombolysis in ischemic stroke. Stroke. (2011) 42:1638-43. doi: 10.1161/STROKEAHA.110.606194

18. Hacke W, Kaste M, Fieschi C, von Kummer R, Davalos A, Meier D, et al. Randomised double-blind placebo-controlled trial of thrombolytic therapy with intravenous alteplase in acute ischaemic stroke (ECASS II).
Second European-Australasian acute stroke study investigators. Lancet. (1998) 352:1245-51. doi: 10.1016/S0140-6736(98)08020-9

19. Chang Y, Kim BM, Bang OY, Baek JH, Heo JH, Nam HS, et al. Rescue stenting for failed mechanical thrombectomy in acute ischemic stroke. A multicenter experience. Stroke. (2018) 49:958-64. doi: 10.1161/STROKEAHA.117.020072

20. Flottmann F, Leischner H, Broocks G, Nawabi J, Bernhardt M, Faizy Tobias D, et al. Recanalization rate per retrieval attempt in mechanical thrombectomy for acute ischemic stroke. Stroke. (2018) 49:2523-5. doi: 10.1161/STROKEAHA.118.022737

21. Hwang YH, Kim YW, Kang DH, Kim YS, Liebeskind DS. Impact of target arterial residual stenosis on outcome after endovascular revascularization. Stroke. (2016) 47:1850-7. doi: 10.1161/STROKEAHA.116.013046

22. Zinkstok SM, Roos YB. Early administration of aspirin in patients treated with alteplase for acute ischaemic stroke: a randomised controlled trial. Lancet. (2012) 380:731-7. doi: 10.1016/S0140-6736(12)60949-0

23. Papanagiotou P, Haussen DC, Turjman F, Labreuche J, Piotin M, Kastrup A, et al. Carotid stenting with antithrombotic agents and intracranial thrombectomy leads to the highest recanalization rate in patients with acute stroke with tandem lesions. JACC Cardiovasc Interv. (2018) 11:1290-9. doi: 10.1016/j.jcin.2018.05.036

24. Wallocha M, Chapot R, Nordmeyer H, Fiehler J, Weber R, Stracke CP. Treatment methods and early neurologic improvement after endovascular treatment of tandem occlusions in acute ischemic stroke. Front Neurol. (2019) 10:127. doi: 10.3389/fneur.2019.00127

25. Garcia-Tornel A, Requena M, Rubiera M, Muchada M, Pagola J, Rodriguez-Luna D, et al. When to stop. Stroke. (2019) 50:1781-8. doi: 10.1161/STROKEAHA.119.025088

Conflict of Interest: JF received research support from German Ministry of Science and Education (BMBF), German Ministry of Economy and Innovation (BMWi), German Research Foundation (DFG), European Union (EU), Hamburgische Investitions- und Förderbank (IFB), Medtronic, Microvention, Philips, Stryker, Consultant for: Acandis, Boehringer Ingelheim, Cerenovus, Covidien, Evasc Neurovascular, MD Clinicals, Medtronic, Medina, Microvention, Penumbra, Route92, Stryker, Transverse Medical. GT received consulting fees from Acandis, grant support and lecture fees from Bayer, lecture fees from Boehringer Ingelheim, Bristol-Myers Squibb/Pfizer, and Daiichi Sankyo, and consulting fees and lecture fees from Stryker. LK received speaker honoraria from Boehringer Ingelheim, Medtronic and JR is consultant for Acandis and Phenox. TA is a consultant for Ablynx, Amnis Therapeutics, Medtronic, Cerenovus/J\&J, Rapid Medical and Anaconda. LY has received substantial grant funding from the National Medical Research Council (NMRC), Singapore and substantial support from the ministry of health $(\mathrm{MOH})$, Singapore. CK is proctor for Acandis. FD is consultant for Acandis. RC is consultant and/or proctor for BALT, Stryker, Microvention, Rapid Medical, Siemens Medical Systems. CS is consultant and/or proctor for Acandis, Balt, and Rapid Medical.

The remaining authors declare that the research was conducted in the absence of any commercial or financial relationships that could be construed as a potential conflict of interest.

Copyright $\odot 2020$ Meyer, Fiehler, Thomalla, Krause, Lowens, Rothaupt, Kim, Heo, Yeo, Andersson, Kabbasch, Dorn, Chapot, Stracke and Hanning. This is an openaccess article distributed under the terms of the Creative Commons Attribution License (CC BY). The use, distribution or reproduction in other forums is permitted, provided the original author(s) and the copyright owner(s) are credited and that the original publication in this journal is cited, in accordance with accepted academic practice. No use, distribution or reproduction is permitted which does not comply with these terms. 